\title{
Linx
}

Revue des linguistes de l'université Paris X Nanterre

\section{L'affaire des paillotes corses, ou le festival de la performativité dévoyée : aveux truqués, dénégations mal récusées, ordres implicites}

Michel Arrivé

\section{(2) OpenEdition \\ Journals}

Édition électronique

URL : http://journals.openedition.org/linx/192

DOI : $10.4000 / \operatorname{linx} .192$

ISSN : 2118-9692

Éditeur

Presses universitaires de Paris Nanterre

Édition imprimée

Date de publication : 1 juin 2005

Pagination : 73-83

ISSN : 0246-8743

\section{Référence électronique}

Michel Arrivé, «L'affaire des paillotes corses, ou le festival de la performativité dévoyée : aveux truqués, dénégations mal récusées, ordres implicites », Linx [En ligne], 52 | 2005, mis en ligne le 27 janvier 2011, consulté le 04 mai 2019. URL : http://journals.openedition.org/linx/192 ; DOI : 10.4000/ linx.192 


\title{
L'affaire des paillotes corses, ou le festival \\ de la performativité dévoyée : aveux truqués, dénégations mal récusées, ordres implicites
}

\author{
Michel Arrivé \\ MoDyCo, UMR 7114 - Université Paris X - Nanterre
}

L'affaire des paillotes corses reste sans doute présente à la mémoire des lecteurs français qui suivent, peu ou prou, l'actualité. Mais rien n'empêche un linguiste de se désintéresser totalement des faits divers, même quand ils ont d'évidentes incidences politiques. Rien n'empêche d'autre part que ce texte soit lu par des étrangers, qui peuvent n'avoir jamais entendu parler de cette ténébreuse affaire. C'est pourquoi je juge utile d'en donner un bref aperçu chronologique, fondé sur le rappel fourni par Le Monde le 13 octobre 2004.

Le 6 février 1998, le préfet de Corse Claude Érignac est assassiné par un groupe d'« indépendantistes ». Dès le 11 février, le Ministre de l'Intérieur, Jean-Pierre Chevènement, nomme Bernard Bonnet pour le remplacer. Le nouveau préfet a acquis dans ses précédents postes la réputation de faire respecter la loi par des méthodes énergiques, parfois même, murmure-t-on, brutales ou retorses. Il se trouve notamment confronté au problème des "paillotes » : restaurants installés de façon illégale sur les plages. En dépit d'injonctions insistantes, les tenanciers refusent de démonter leurs installations. Dans la nuit du 19 au 20 avril 1999, une paillote est détruite par un incendie. Les soupçons se portent sur les gendarmes, qui expliquent leur geste en alléguant qu'ils l'ont commis sur l'ordre du préfet. Le procès des responsables 
présumés - notamment le préfet Bonnet et le colonel de gendarmerie Mazères - a lieu en décembre 2001 et janvier 2002. Le préfet s'obstine à dénier toute responsabilité dans l'incendie. Le 11 janvier 2002, le procès s'achève par la condamnation du préfet à trois ans de prison, dont deux avec sursis. Le colonel Mazères est condamné à deux ans et demi de prison, dont deux avec sursis.

Le mardi 12 octobre 2004, à la veille du procès en Cassation, Le Monde publie en première page, dans son édition datée du lendemain mercredi 13, un article intitulé : "Affaire des paillotes corses : les aveux du préfet Bonnet». En page intérieure, un autre article porte pour titre : "Bernard Bonnet a donné l'“ordre implicite" de brûler les paillotes ». Dans son édition du lendemain (publiée le 13, datée du 14), Le Monde présente l'interprétation du colonel de gendarmerie, sous le titre : « Le colonel Mazères maintient que l'ordre de brûler les paillotes était explicite et répété ».

Le mercredi 13 octobre, la Cour de cassation confirme les condamnations du préfet Bonnet et du colonel Mazères. L'affaire, au moment (fin octobre 2004) où ces lignes sont écrites, n'est sans doute pas terminée. Le préfet Bonnet a demandé la grâce présidentielle. Compte tenu des « aveux » du préfet, les avocats du colonel Mazères envisagent (Le Monde du 14/10/04) de demander que l'affaire soit rejugée.

Les deux articles successifs du Monde fournissent à foison des exemples d'énoncés performatifs, dans des conditions rendues particulièrement intéressantes à la fois par la situation référentielle, spécialement embrouillée, et par l'enjeu que comportent ces énoncés : rien de moins que la carrière et la liberté des deux principaux intéressés. Sans parler des graves problèmes posés par l'implication du pouvoir politique dans un fait divers d'allure assez crapuleuse : c'est que le préfet est le représentant du gouvernement dans les départements ou les régions, et la gendarmerie est son bras armé. Indice non trompeur de cette implication du pouvoir, au plus haut niveau, dans l'affaire : le ministre responsable de la nomination du préfet Bonnet se trouve amené à prendre la parole dans l'un des articles du Monde.

Les trois types d'énoncés qui émergent relèvent - sous des formes, on le verra, fortement spécifiques - de l'aveu, de la dénégation et de l'ordre.

L'aveu. C'est le titre même de l'article en première page du Monde du 13/10/04. Conformément à un usage fréquent, le nom aveu, qui n'est pas placé entre guillemets, est pluralisé. Il paraît peu vraisemblable, on s'en rendra compte dans la suite, que le rédacteur vise plusieurs actes d'aveu : c'est tout au plus s'il parvient, non sans peine, à en repérer un. Son pluriel est sans doute le "pluriel interne », fréquent avec ce nom, par exemple dans l'expression figée passer aux aveux.

Quoi qu'il en soit, l'«aveu » du préfet prend une forme particulière. La demande formulée par le rédacteur du Monde est, sans l'ombre d'une quelconque ambiguïté, une demande d'« aveu» :

Après l'avoir toujours niée, reconnaissez-vous, aujourd'hui, votre responsabilité dans l'affaire? 
Mais le préfet feint de ne pas entendre cette demande. Au bref énoncé performatif attendu il substitue un long énoncé strictement constatif, du type du récit, même si le préfet recourt occasionnellement au présent de narration :

Il ne s'agit pas d'éluder des erreurs ou des responsabilités, mais d'essayer de comprendre ce qui s'est passé. (...). Tout s'écroule alors. Depuis cette date [celle de la mise en garde à vue du préfet dans son bureau, MA], il y a eu de ma part un raidissement, et des postures à contre-emploi (...).

Le journaliste n'a pas obtenu l'aveu demandé : il ne se contente pas du récit qui lui est offert. Il s'obstine, et réitère, sous la forme la plus directe, sa demande d'aveu :

Avez-vous donné l'ordre d'incendier ces paillotes?

Le préfet est poussé dans ses derniers retranchements, et en vient à ce qui sera considéré par Le Monde comme un « aveu » et lui fournira le titre de son article :

Il s'agissait d'un ordre implicite, mais non formalisé.

On aperçoit la stratégie du préfet : avec une maladresse presque pathétique, il essaie de limiter les dégâts. Sa stratégie discursive est double. D'une part, il reste dans le registre de l'énonciation historique, qui lui permet à la fois de demeurer dans le passé et d'éviter, en même temps que la première personne, la performativité au sens strict, indispensable à l'acte même de l'aveu: l'événement est rapporté de façon impersonnelle par la formule «il s'agissait de ». Il semble ainsi s'être déroulé de luimême, sans intervention intentionnelle de la part du préfet ${ }^{1}$. D'autre part il introduit la notion d'« ordre implicite », qui lui permettra - on le verra un peu plus loin - d'aller jusqu'à se demander s'il y a eu véritablement un «ordre», et de ce fait s'il y a réellement quelque chose à avouer.

D'une certaine façon, Jean-Pierre Chevènement - qui a eu en tant que Ministre de l'Intérieur la responsabilité de la nomination du préfet Bonnet - a presque raison (dans Le Monde du 14/10/04) de dire qu'« il n'y a rien de nouveau sous le soleil » et d'attribuer au « génie des titres du journal Le Monde » la qualification d'« aveux » conférée aux propos du préfet. Certes, la stratégie du préfet est à la fois retorse et naïve : à aucune des étapes du procès les magistrats ne s'y seront trompés. Mais il reste exact que, même dans les propos rapportés par le journal, il n'y a pas eu d'aveu au sens formel du terme, et que les faits malgré tout reconnus à mi-voix peuvent, selon une lecture hyper-littérale, ne pas être considérés comme des « ordres ». Faut-il préciser que l'attitude de lecture hyper-littérale de l'ancien ministre ne tient nullement à des scrupules d'exactitude philologique ni à des conceptions linguistiques longuement

\footnotetext{
${ }^{1}$ On aura reconnu, avec sa terminologie, l'appareil conceptuel mis en place par Benveniste dans 1959-1966 (pour l'opposition des deux registres de l'bistoire et du discours) et 1963-1966 (pour sa conception spécifique de la performativité, voir notamment p. 274). On se souvient que Benveniste tient, contrairement à Austin (1970) et à ses continuateurs, pour une conception étroite de la performativité, qui en limite l'extension aux énoncés comportant un verbe au présent et à la première personne. Un énoncé tel que « il s'agissait d'un ordre » ne peut en rien, dans l'optique de Benveniste, être considéré comme performatif, et ne constitue donc pas un aveu.
} 
méditées, mais au souci plus terre-à-terre de dégager totalement sa propre responsabilité dans l'affaire?

La dénégation non récusée. Le préfet, assez justement, donne le nom de «dénégation» à l'attitude énonciative qu'il a adoptée avant d'en venir, dans les conditions qui viennent d'être rapportées, au faux-semblant d'aveu qui fait le titre du journal. Il concède qu'il a longtemps dénié toute forme de responsabilité dans l'incendie des paillotes. Dans le récit qu'il fait des événements passés, il est naturellement contraint d'utiliser la première personne. Mais c'est une première personne désormais évanouie dans un passé explicitement aboli :

Avec le recul, j'ai choisi à cette époque un système de défense illisible qui s'est révélé cristalliser le conflit, et finalement inefficace. Je me suis enfermé dans ce système, je me suis séquestré dans la dénégation, et cela a provoqué chez moi un comportement que je considère comme éloigné de l'éthique que je me suis toujours faite d'un préfet républicain.

Le journaliste du Monde voit clairement l'importance de cette dénégation passée. Si le préfet la récuse, il reconnaîtra sa responsabilité : l'aveu toujours — ou de nouveau - attendu sera fourni de façon négative. Le journaliste formule donc sa demande de récusation. Mais il le fait, d'une façon sans doute maladroite, sous la forme d'une question historique :

Pourquoi vous êtes-vous arc-bouté sur des dénis ?

Le préfet est évidemment ravi de l'occasion qui lui est donnée de ne pas quitter l'histoire. Il réitère donc, au présent de narration, sa récusation, en lui conférant une explication simultanément historique, psychologique et juridique :

Je vous l'ai dit, le 3 mai $1999^{2}$ je suis tétanisé. Ma première défense, c'est de penser qu'il n'y a pas d'infraction pénale, que tout cela n'est pas grave. Je minimise.

En ce point le préfet n'a pas vraiment avoué et n'a même pas récusé la dénégation qu'il a formulée. Précisons-le : contrairement à ce qu'ont pu faire d'autres accusés dans des circonstances comparables, il n'a à aucun moment réussi à être adroit. La fin de l'histoire - sa condamnation à un an de prison ferme - le lui fera savoir cruellement. Mais il a au moins tenté de manipuler toutes les possibilités dont il pouvait disposer pour essayer de masquer cet obscur objet qui hante le discours des hommes : le référent, sous la forme, ici, du donné des événements ${ }^{3}$.

Ce donné événementiel, finalement, se réduit à un acte. Un acte qui est à la fois, indissolublement, un acte de langage et un acte extralinguistique, en prise directe sur le

\footnotetext{
${ }^{2}$ C'est à cette date que le préfet et le directeur de son cabinet Gérard Pardini sont placés en garde-àvue dans leurs bureaux de la Préfecture.

${ }^{3}$ Je précise cependant qu'à un autre moment de l'entretien - quand on ne lui demande pas de le faire... - le préfet frôle de très près la récusation de sa dénégation. Sans toutefois la réaliser pleinement. Il se contente en effet de la rejeter dans le passé : «Moi, j’avais pris la très mauvaise décision de m’installer dans la stratégie, éprouvante, de la dénégation ».
} 
réel : un ordre ${ }^{4}$. Cet ordre a-t-il été donné ? Autrement dit, y a-t-il quelque chose à avouer ? C'est ici qu'intervient la notion d'« ordre implicite ».

L'ordre implicite. On vient d'apercevoir que lors de son « aveu » le préfet reconnaît l'existence "d'un ordre implicite, non formalisé ». Il reprend exactement cette formule - « un ordre implicite, non formalisé » - dans la suite de l'entretien. En un autre point, il dit, cette fois dans une tournure impersonnelle, qu'« il n'y a pas d'ordre formel ».

Il est facile de voir ce que le préfet entend par l'adjectif formalisé et sa variante formel. Contrairement à ce qu'on pouvait attendre, ce n'est pas le sens administratif de l'adjectif, mais son sens à strictement parler linguistique. L'ordre formalisé ou formel serait celui qui comporterait la forme linguistique conférée à l'expression «littérale » de l'« ordre » : le mode impératif. C'est ce que dit le préfet, dans un récit qui, une fois de plus, recourt au présent de narration :

(...) il n'y a pas d'ordre formel, je ne lui [au colonel, MA] dis pas : « Allez incendier la paillote $»$.

On le voit : le préfet est d'une précision exemplaire sur ce qu'il ne dit pas et, du coup, ne fait pas. Il a sans doute raison : l'ordre littéral, à l'impératif, n'a vraisemblablement pas été donné. Mais hélas, il est moins éloquent sur ce qu'il dit et fait. Il faut lire de près la totalité de l'entretien pour trouver, à un endroit où on ne l'attend pas, la forme précise qui a été conférée à l'« ordre implicite » :

Le 10 avril au soir [jour de l'échec d'une tentative d'expulsion des exploitants, MA], cela se passe très mal avec le colonel Henri Mazères. Je lui dis : «Mon colonel, c’est lamentable, vous sabotez l'opération, on fait d'Yves Féraud [le gérant de la paillote, $\mathrm{MA}$ ] un héros, alors qu'il s'agit d'un hors-la-loi. La prochaine fois, j'irai moi-même sur le terrain ».

Sur ce point, la lettre même des propos de Bonnet est confirmée à la fois par le colonel Mazères et par le propre directeur de cabinet du préfet, Gérard Pardini. Naturellement les deux fonctionnaires sont en désaccord complet avec leur supérieur sur la nature - implicite ou explicite? - de l'ordre ainsi donné : le colonel s'exprime ainsi :

Un jour, il m’a dit : «Vous faites tout de suite chez Francis, sinon j’irai moi-même ».

Et Pardini de confirmer :

C'est quand le préfet a dit qu'il allait le faire lui-même que j'ai agi. C'était une logique de fidélité et de protection. J'ai honte d'avoir obéi dans de telles conditions.

\footnotetext{
${ }^{4}$ Je constate avec une certaine surprise que la notion d'ordre ne donne pas lieu à une entrée dans des dictionnaires (Charaudeau-Maingueneau, 2002 et Moeschler-Reboul, 1994) où on pouvait l'attendre. Inversement, on trouve une entrée pour ce mot dans Dubois et alii, 1994 ainsi que dans Arrivé et alii, 1986. L'ordre apparait, sous les espèces de la requête, dans l'index de Kerbrat-Orecchioni, 1986. L'aven et la dénégation sont également privés d'entrées dans Charaudeau-Maingueneau et Moeschler-Reboul ainsi que dans l'index de Kerbrat-Orecchioni.
} 
Dans la forme complète présentée dans les propos du préfet, l'« ordre implicite » comporte les deux composantes suivantes :

1. La déploration de la non-exécution, présente dans le « c'est lamentable, vous sabotez l'opération ». Cette déploration présuppose évidemment l'énonciation antécédente d'un (ou de plusieurs) ordre(s) préalable(s), malencontreusement non exécutés.

2. La menace de l'exécution substitutive, mise en forme par le «j'irai moi-même». Menace dont l'efficacité se fonde sur la relation hiérarchique entre le préfet et ses deux adjoints. Elle n'aurait eu aucun effet en l'absence de la subordination au préfet de ses deux acolytes. On sait depuis Austin 1970 et Searle 1972 (et, bien sûr, depuis bien longtemps avant...) que ce type de relation est indispensable à la réussite de certains actes de langage, notamment les prescriptifs.

Bien qu'ils soient en plein accord sur la lettre des propos tenus, le préfet et le colonel divergent totalement sur la nature de l'ordre : le préfet tient pour l'implicite, le colonel pour l'explicite et le répétitif. Comment s'explique cette divergence d'appréciation sur le même objet?

Le préfet se fonde sur l'absence de forme directement impérative. Il en viendra même à se demander, avec une hypocrisie qui transparaît dans la forme, de nouveau très contournée, de ses propos, s'il y a eu véritablement un ordre :

Mazères est convaincu qu'il a une incitation à agir. Est-ce un ordre ? (...) Il entend un ordre que je n'ai pas le sentiment de lui avoir formellement donné.

En ce point, pour le préfet, la notion d'ordre se réduit à celle d'ordre « formel», comportant un impératif. En sorte que la notion d'« ordre implicite » qui tient une place centrale au début de sa défense, en vient opportunément à se dissoudre complètement. D'où l'interrogation, à vrai dire très étonnante : "Est-ce un ordre ? ", puis l'effort effectué pour distinguer l'« ordre » (totalement récusé) de l'« incitation à agir » (plus ou moins acceptée). La position est difficile à tenir : le sujet est totalement clivé. D'un côté, il souhaite que l'ordre soit exécuté, de l'autre il regrette qu'il l'ait été, et de ce fait en vient à contester qu'il ait été donné. Le préfet, confusément, prend conscience de ce clivage et le décrit en termes éthiques:

Cela a provoqué chez moi un comportement que je considère comme éloigné de l'éthique que je me suis toujours faite d'un préfet républicain ${ }^{5}$.

Le colonel de son côté tient à la fois que l'ordre a été explicite et répété. Pour la répétition, l'énoncé du préfet lui donne raison, puisqu'il présuppose au moins un « ordre » antécédent non exécuté. Pour le problème de la distinction entre implicite et

\footnotetext{
${ }^{5}$ Le préfet a même été tenté par une solution extrême : laisser entendre qu'en l'absence d'impératif formel, il n'y a eu ni ordre, ni même incitation à agir. Ce qui lui permet de chercher à adopter pour un instant la noble attitude du préfet assumant la responsabilité entière de tous les actes, même non prescrits, de ses subordonnés : «Quand on est préfet, on assume les réussites, les échecs, les excès de ceux que vous dirigez ». Cependant les difficultés de cette posture pour lui acrobatique sont telles qu'il l'abandonne aussitôt : la belle figure du "préfet républicain » qui assume indifféremment échecs et réussites de ses subordonnées n'est pas à sa portée.
} 
explicite, il semble bien que le gendarme fonde son interprétation sur l'efficacité même de l'ordre, sur l'" obéissance », selon le mot de Pardini. Comme on l'a vu, il tombe en effet en accord avec le préfet sur la lettre des propos tenus :

Cet ordre lui «a été donné formellement, à plusieurs reprises, et répété une ultime fois sous la forme: "si vous ne le faites pas, c'est mois (sic) qui le ferai" ».

C'est donc qu'il tient la formule du préfet pour un " ordre explicite ». Il a en effet une conception très extensive de la «formalité » de l'ordre. Contrairement à son supérieur, il accepte la notion d'acte de langage indirect, et reconnait aux formulations indirectes de l'ordre un statut comparable à leurs équivalents formalisés. Il en vient finalement à neutraliser, au moins pour l'ordre, la distinction de l'implicite et de l'explicite. Relève de l'ordre, selon lui, tout énoncé dont la profération entraîne l'« obéissance », c'est-à-dire la réalisation d'un acte, quelle que soit la forme, littérale ou figurée, implicite ou explicite, dénotée ou connotée, etc., qui se trouve affectée à la désignation de cet acte. On le voit: le préfet et le colonel ont en matière de performativité des conceptions absolument opposées. Le colonel est proche d'Austin et Searle dans leurs conceptions extensives, le préfet n'est pas loin de rejoindre Benveniste dans sa position ascétique.
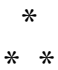

On l'a certainement repéré : l'affaire des paillotes corses a donné lieu à un véritable festival de la performativité. Par souci d'échapper à leurs responsabilités, les deux acteurs principaux se laissent aller à des manipulations qui couvrent un spectre extrêmement étendu. Ces manipulations portent sur les énoncés performatifs euxmêmes : on aura apprécié la variété des exemples d'aveu, de dénégation et d'ordre qui sont fournies par le corpus pourtant bien mince qui a été exploité. Mais elles atteignent aussi les énoncés métaperformatifs: on trouve chez le préfet et le colonel deux véritables théories, à peine sous-jacentes, de la performativité. Théories qui, on ne s'en étonne point, sont à la fois opposées et, surtout chez le préfet, évolutives : c'est qu'elles sont déterminées exclusivement par le souci, d'ailleurs mal compris, de leur efficacité pour leur défense.

Performativité dévoyée, ai-je cru pouvoir écrire dans le titre de ce bref article. C'est au terme de l'étude qu'il convient de se poser une question : par rapport à quoi la performativité est-elle ici dévoyée?

Serait-ce que les discours du préfet et du colonel s'écartent des possibilités offertes par le langage? Que non pas : leurs propos sont d'une normalité linguistique absolue. Ils n'ont jamais étonné le journaliste qui les a écoutés. Quant aux fragments de métadiscours qu'ils se laissent aller à émettre, ils se répartissent sans difficulté dans l'inventaire des opinions communément émises par les linguistes spécialistes du problème.

Serait-ce donc par rapport à l'éthique que ces propos sont dévoyés ? Le préfet lui-même semble bien avoir un début d'inquiétude à cet égard : on a vu plus haut qu'il en vient, fugitivement, à évoquer « l'éthique d'un préfet républicain ». Toutefois cette allusion à l'éthique vise exclusivement les actes qu'il a effectués, et non les spécificités 
de son discours. C'est que l'éthique a avec le langage des relations complexes. Les linguistes, longtemps, n'ont pas abordé ce problème. Hjelmslev est sans doute l'un des premiers à l'envisager : après avoir posé la propriété qu'ont les langues de traduire non seulement toute langue, mais encore toute « sémiotique concevable ${ }^{6}$ ", il explique cette propriété de la façon suivante :

Nous sommes enclin à supposer que la raison en est la possibilité illimitée de formation des signes et les règles très libres qui régissent la formation d'unités de grande étendue (comme les phrases par exemple) dans toutes les langues, ce qui, d'autre part, a pour effet de permettre des formulations fausses, illogiques, imprécises, laides et immorales (en danois : uetiske) aussi bien que des formulations vraies, logiques, précises, belles et morales (en danois : etiske). Les règles grammaticales d'une langue sont indépendantes de toute échelle de valeurs, qu'elle soit logique, esthétique ou éthique (en danois de nouveau etisk, ce qui aurait donc dû imposer les traductions non éthiques et éthiques pour les occurrences précédentes de l'adjectif) et, de façon générale, la langue est dépourvue de toute finalité (1943, p. 97, 1971, p. 138).

On ne peut qu'approuver cette analyse, pour laquelle il n'est que trop aisé de trouver des illustrations frappantes, tant dans le domaine des signes (la langue, ou, en termes hjelmsléviens, le système) que dans celui des « unités de grande étendue » (les phrases et le discours, ou, en termes hjelmsléviens, le procès).

Il convient cependant, en limitant ici le problème à ses aspects éthiques, de formuler deux importantes réserves :

1. C'est la formation des unités linguistiques qui échappe à toute loi éthique. Mais après leur formation, le caractère éventuellement «non éthique » des signes et des discours tombe sous le coup d'un jugement éthique, qui peut dans certains cas être formalisé sous l'aspect judiciaire. Pour les « signes » (c'est-à-dire les mots), il existe des exemples (rares et suivis de peu d'effets) de tentatives d'épuration du lexique à visée éthique : c'est que les mots ont la vie dure, et persistent en dépit des interdictions ou des stigmatisations. Que faire pour qu'un mot chargé d'un long passé n'existe plus, ou, mieux encore, n'ait jamais existé ? Le problème se pose pour un grand nombre de désignations ethniques ou religieuses à valeur péjorative ou injurieuse: qu'on se reporte par exemple aux articles arabe, juif ou turc dans les éditions successives du Dictionnaire de l'Académie française ${ }^{7}$. Ou encore, qu'on relise Molière (notamment L'Avare

\footnotetext{
${ }^{6}$ Faut-il rappeler que la traduction de 1971, contrairement à celle de 1968, utilise le terme sémiotique pour traduire, d'ailleurs de façon non constante, le sprog danois de Hjelmslev, dont la traduction habituelle est langage? La raison de ce choix teint sans doute dans la nécessité de distinguer commodément langue (en hjelmslévien dagligsprog) de langage (sprog).

${ }^{7}$ Encore en 1835, le mot juif « se dit aussi, figurément et familièrement, de celui qui prête à usure ou qui vend exorbitamment cher, et en général de quiconque cherche à gagner de l'argent par des moyens injustes et sordides ». Cette formulation aggrave lourdement celle de 1694, ou le juif se contente d'être « un homme qui preste à usure ». L'édition de 1932 atténue très légèrement celle de 1835 : «Celui qui prête à usure et qui se conduit, en affaires, avec avidité et âpreté ». Et il faut attendre la 9ème édition (1992) pour voir la valeur péjorative reléguée dans les oubliettes de l'histoire :
} 
et Les fourberies de Scapin), pour voir quel sens est conféré, au milieu du XVII siècle, à ces trois mots.

Pour les discours, il semble plus facile d'agir. L'exemple du négationnisme est celui qui parait le plus pertinent. Comme son nom suffit à l'indiquer, le négationnisme est un phénomène discursif, décrit avec pertinence par Alain Bihr :

Le négationnisme consiste dans la négation pure et simple de l'entreprise génocidaire nazie: au cours de la Seconde Guerre mondiale, le régime nazi n’aurait pas développé une politique visant à l'extermination systématique, sous différentes formes, de certaines catégories des populations placées sous sa domination, identifiées sur la base de critères raciaux et/ou politiques (1997, p. 125).

C'est ce type de discours, quand il est public (mais à vrai dire tout discours, sauf le monologue intérieur ${ }^{8}$, n'est-il pas plus ou moins public ?) qui est visé par les dispositions de la « loi Gayssot »:

Art. 24 bis. (L. n. 90-615, 13 juill, 1990, art. 9). - Seront punis des peines prévues par le sixième alinéa de l'article 24 ceux qui auront contesté, par un des moyens énoncés à l'article 23, l'existence d'un ou plusieurs crimes contre l'humanité tels qu'ils sont définis par l'article 6 du statut du tribunal militaire international annexé à l'accord de Londres du 8 août 1945 et qui ont été commis soit par les membres d'une organisation déclarée criminelle en application de l'article 9 dudit statut, soit par une personne reconnue coupable de tels crimes par une juridiction française ou internationale ; [...]

2. Les énoncés performatifs. Il semble qu'ils ne soient en rien pris en compte par Hjelmslev. Non qu'il se désintéresse des problèmes de l'énonciation : j’ai cru montrer (Arrivé 1986) que, contrairement à une opinion communément reçue, il existe bien en glossématique une théorie, à vrai dire très spécifique, de l'énonciation. Mais des performatifs, sous ce nom ou sous un autre, il n'est, si j'ai bien lu, jamais question. Qu'on s'en étonne ou non - car Hjelmslev, une fois de plus à l'inverse de sa réputation, s'intéresse aux effets du discours ${ }^{9}-$ le fait est là. Il n'a donc pas l'occasion de remarquer cette évidence: les énoncés performatifs, qui constituent des actes, échappent par là-même à la non-éthicité des unités linguistiques. Ils tombent nécessairement d'emblée sous le coup des lois éthiques. Il conviendrait donc d'élaborer une éthique de la performativité. Il va sans dire que je ne me lancerai pas

«HIST. Au Moyen Âge, nom donné aux prêteurs à usure, parce que seuls les Juifs étaient autorisés, avec les Lombards, à pratiquer ce métier. » Reste à savoir si le mot reste bien enfermé dans les oubliettes où les Académiciens l'ont très judicieusement enfermé...

${ }^{8}$ Sur les problèmes terminologiques concernant les divers phénomènes d'endophasie, voir Bergounioux, 2004.

$9^{9}$ «Le système des signes, le langage et la forme du contenu sont devenus une force qu'aucun dirigeant ne peut ou ne veut négliger d'utiliser. [...] Celui qui a le désir et le moyen de le faire met en mouvement la volonté des masses non seulement en utilisant des mots et des gestes, mais aussi des symboles tels que la svastika ou la faucille et le marteau. [...] Quiconque aspire à être dictateur ferait bien d'étudier la sémantique » (1953, in 1971 b, p. 101). 
dans le travail de cette élaboration. Je me laisserai seulement aller à remarquer que les discours du préfet et du colonel ne donnent sans doute pas d'excellents exemples de performativité éthique.

Resterait à poser un ultime problème. C'est en tant qu'actes que les énoncés performatifs sont soumis à l'éthique. Comment des énoncés apparemment non performatifs peuvent-ils également y être soumis ? Une négation pourrait-elle autre chose qu'un énoncé constatif? Autrement dit, comment se justifie l'application de la loi Gayssot au discours négationniste? En juriste, Michel Troper nous fournit une réponse :

Par la loi Gayssot, le Parlement a donc institué une présomption. En punissant la négation du génocide des mêmes peines que l'incitation à la haine raciale, il présume qu'elle est un acte équivalent parce qu'il est de même nature et qu'il porte comme lui atteinte à des intérêts qui doivent être protégés. (1999).

Le juriste pose sans hésitation la nature d'acte du discours négationniste. Il le ferait de la même façon, en d'autres circonstances, pour un énoncé affirmatif présentant le même caractère d'« incitation à la haine raciale ». Il rencontre ici (sans le savoir? En tout cas il n'y fait pas allusion) un problème qui a longuement occupé linguistes et philosophes: celui de l'éventuelle performativité des énoncés apparemment constatifs. Je ferai ici comme Troper : je garderai le silence. 


\section{BIBLIOGRAPHIE}

ArrivÉ, M. (1986), "Y a-t-il en glossématique une théorie de l'énonciation ?», Histoire, épistémologie, langage, VIII-2, p. 177-189.

ARrivÉ, M., Gadet, F., Galmiche, M. (1986), La grammaire d'aujourd'bui, guide alphabétique de linguistique française, Paris, Flammarion.

Austin, J. (1970), Quand dire, c'est faire, Paris, Le Seuil.

Benveniste, É. (1959-1966), "Les relations de temps dans le verbe français », in Bulletin de la Société de Linguistique de Paris, t. LIV, fascicule 1. Repris dans Benveniste, 1966, p. 237 250.

BENVEnISTE, É. (1963-1966), «La philosophie analytique et le langage », in Les Études philosophiques, $\mathrm{n}^{\circ} 1$, janvier-mars 1963. Repris dans Benveniste, 1966, p. 267-276.

Benveniste, É . (1966), Problèmes de linguistique générale, Paris, Gallimard.

Bergounioux, G. (2004), Le moyen de parler, Lagrasse, Verdier.

BiHr, A. (1997), "Les mésaventures du sectarisme révolutionnaire», in Négationnistes : les chiffonniers de l'bistoire, Paris, Éditions Golias et Éditions Syllepse.

Charaudeau, P. et Maingueneau, D. (2002), Dictionnaire d'analyse du discours, Paris, Le Seuil.

DubOIS, J. et alii (1994), Dictionnaire de linguistique et des sciences du langage, Paris, Larousse.

HJelmslev, L. (1943) (1971 a), Prolégomènes à une théorie du langage, Paris, Éditions de Minuit.

HJelmsLeV, L. (1953), « La forme du contenu du langage comme facteur social ». Repris dans 1971 b, p. 97-104.

HJelmslev, L. (1971 b), Essais linguistiques, Paris, Éditions de Minuit.

Kerbrat-Orecchionni, C. (1986), L’implicite, Paris, Armand Colin.

Moeschler, J. et Reboul, A. (1994), Dictionnaire encyclopédique de pragmatique, Paris, Le Seuil.

SEArLe, J. R. (1972), Les Actes de langage, Paris, Hermann.

Troper, M. (1999), « La loi Gayssot et la constitution », Annales, Histoire, Sciences Sociales, 54 (6), novembre-décembre. 
